\title{
The properties of SN Ib/c locations ${ }^{\star}$
}

\author{
G. Leloudas ${ }^{1}$, A. Gallazzi ${ }^{1}$, J. Sollerman ${ }^{2}$, M. D. Stritzinger ${ }^{2}$, J. P. U. Fynbo ${ }^{1}$, J. Hjorth ${ }^{1}$, D. Malesani ${ }^{1}$, \\ M. J. Michałowski ${ }^{3}$, B. Milvang-Jensen ${ }^{1}$, and M. Smith ${ }^{4}$ \\ 1 Dark Cosmology Centre, Niels Bohr Institute, University of Copenhagen, Juliane Maries Vej 30, 2100 Copenhagen $\emptyset$, Denmark \\ e-mail: giorgos@dark-cosmology.dk \\ 2 The Oskar Klein Centre, Department of Astronomy, Stockholm University, AlbaNova, 10691 Stockholm, Sweden \\ 3 Scottish Universities Physics Alliance, Institute for Astronomy, University of Edinburgh, Royal Observatory, Edinburgh, \\ EH9 3HJ, UK \\ 4 Astrophysics, Cosmology and Gravity Centre, Department of Mathematics and Applied Mathematics, University of Cape Town, \\ Cape Town 7700, South Africa
}

Received 10 February 2011 / Accepted 7 March 2011

\section{ABSTRACT}

\begin{abstract}
Aims. We seek to gain a deeper understanding of stripped-envelope, core-collapse supernovae through studying their environments. Methods. We obtained low-resolution optical spectroscopy with the New Technology Telescope (+EFOSC2) at the locations of 20 type $\mathrm{Ib} / \mathrm{c}$ supernovae. We measured the flux of emission lines in the stellar-continuum-subtracted spectra from which local metallicities are computed. For the supernova regions, we estimate both the mean stellar age, by interpreting the stellar absorption with population synthesis models, and the age of the youngest stellar populations using the $\mathrm{H} \alpha$ equivalent width as an age indicator. These estimates are compared with the lifetimes of single massive stars.

Results. Based on our sample, we detect a tentative indication that type Ic supernovae might explode in environments that are more metal-rich than those of type Ib supernovae (average difference of $0.08 \mathrm{dex}$ ), but this is not a statistically significant result. The lower limits placed on the ages of the supernova birthplaces are generally young, although there are several cases where these appear older than what is expected for the evolution of single stars that are more massive than 25-30 $M_{\odot}$. This is only true, however, when assuming that the supernova progenitors were born during an instantaneous (not continuous) episode of star formation.

Conclusions. These results do not conclusively favor any of the two evolutionary paths (single or binary) leading to stripped supernovae. We do note a fraction of events for which binary evolution is more likely due to their associated age limits; however, the supernova environments contain areas of recent $(<15 \mathrm{Myr})$ star formation, and the environmental metallicities at least do not contradict the single evolutionary scenario, suggesting that this channel is also broadly consistent with the observations.
\end{abstract}

Key words. supernovae: general - stars: evolution - galaxies: abundances

\section{Introduction}

The progenitors of stripped-envelope core-collapse (CC) supernovae (SNe) still evade direct detection (Smartt 2009), although reasoning dictates that they are Wolf-Rayet (WR) stars (Crowther 2007). This class of explosions encompasses the hydrogen-deficient supernovae of types Ib and Ic (collectively $\mathrm{SNe} \mathrm{Ib} / \mathrm{c}$ ), but also SNe IIb that retain part of their hydrogen envelope. In Leloudas et al. (2010) we showed that the locations of $\mathrm{SNe} \mathrm{Ib} / \mathrm{c}$ within their host galaxies are indeed consistent with those of WR stars. Several open questions exist in relation to these stellar explosions, the most fundamental of which probably is whether they result from the evolution of single or binary stars.

To appear as a $\mathrm{SN} \mathrm{Ib/c,} \mathrm{the} \mathrm{progenitor} \mathrm{star} \mathrm{has} \mathrm{to} \mathrm{undergo}$ significant stripping of its outer hydrogen envelope. This could be the result of mass loss due to stellar winds in single massive stars. Alternatively, the outer envelope could be shed owing to binary evolution (e.g. Podsiadlowski et al. 1992). The idea that at least a significant fraction of stripped-envelope $\mathrm{SNe}$ come from a binary channel presently enjoys significant

* Based on observations collected at the European Southern Observatory during ESO Program 081.D-0757(A). support (e.g. Fryer et al. 2007; Smartt 2009; Smith et al. 2011), based largely on arguments related to the stellar initial mass function (IMF) and the observed ratio of type Ib/c to type II SNe.

Valuable insight into the nature of these explosions can be gained through observations of their environments because the environmental properties can be used for a number of tests. In the single massive star evolutionary scenario, normal SNe Ic are expected to arise in higher metallicity environments than $\mathrm{SNe} \mathrm{Ib}$. This is because wind-driven mass loss for single massive stars depends strongly on metallicity (Vink \& de Koter 2005), and $\mathrm{SNe}$ Ic need to lose more mass than $\mathrm{SNe} \mathrm{Ib}$. If the mass loss is instead caused by binary evolution, it is probably independent of metallicity (Fryer et al. 2007). Therefore, determining the metallicity at the locations of type $\mathrm{Ib} / \mathrm{c} \mathrm{SNe}$ has the potential of probing their origins. Another potential probe of the explosion progenitor is to determine the age of the local stellar population. This is because single massive stars should only be found in young, actively star-forming regions. For such environmental studies, large samples are necessary for a statistically reliable approach.

Although indirect (Prantzos \& Boissier 2003) and direct (Prieto et al. 2008) global host galaxy metallicity measurements exist, studies focusing on the local environments have been 
based until recently on proxies of metallicity (Anderson \& James 2009; Boissier \& Prantzos 2009). Two contemporary studies (Anderson et al. 2010; Modjaz et al. 2011) report on SN host galaxy metallicity measurements that are both local (at the site of the SN) and direct (using local emission-line metallicity estimates). Anderson et al. (2010) studied 74 host H II regions of $\mathrm{CC} \mathrm{SNe,} \mathrm{of} \mathrm{which} 27$ were type Ib/c SNe, while the Modjaz et al. (2011) study focuses on $35 \mathrm{SNe} \mathrm{Ib} / \mathrm{c}$. The two studies disagree somewhat regarding the existence of differences in the environmental metallicities between type $\mathrm{Ib}$ and type Ic SNe. Anderson et al. (2010) find equal metallicities between the two, while Modjaz et al. (2011) report on a difference of 0.20 dex, which they argue is statistically significant.

In this paper we report on similar observations of $20 \mathrm{SNe} \mathrm{Ib/c}$ sites (15 of which are new), thereby increasing the existing sample. Our targets, observations, and data reduction are presented in the next section, where we also discuss the critical issue of spectroscopic classifications. In Sect. 3 the methods for subtracting the stellar continuum from the spectra, measuring the local metallicities at the SN sites and estimating the age of the local stellar populations are described. Section 4 contains a discussion of our results and Sect. 5 concludes our study.

\section{The data and data reductions}

We targeted host galaxies of normal stripped-envelope CC SNe that have been closely monitored by either the Carnegie Supernova Project (CSP) or the SDSS-II Supernova Survey. For the present study the sample selection is only relevant for understanding the potential biases in our data.

The CSP (Hamuy et al. 2006) obtained unprecedented photometry and spectra for $\sim 250 \mathrm{SNe}$ of all types, 35 of which were stripped $\mathrm{CC} \mathrm{SNe}$. These $\mathrm{SNe}$ were discovered by different search programs that typically monitor bright $\left(m_{\mathrm{B}} \sim 11-16\right)$ nearby galaxies. This, of course, introduces an unavoidable selection bias.

The SDSS-II Supernova Survey (Frieman et al. 2008) scanned an equatorial strip of the sky searching for SNe Ia to be used for cosmology (see Kessler et al. 2009). Other types of SNe were also discovered, including 20 stripped-envelope CC SNe. The SDSS SN host galaxies are typically fainter $\left(m_{\mathrm{g}} \sim 17-22\right)$ and less spatially resolved than those followed by the CSP. Spectra for some of the brightest of these galaxies exist in the SDSS database (e.g. Prieto et al. 2008), but these spectra are centered on the galaxy nucleus and not on the SN location itself. The SDSS sample is a relatively unbiased sample from a host galaxy point of view. We note, however, that this survey was targeting mainly SNe Ia and that the selection of CC SNe was not made in order to obtain a complete, unbiased sample. Some CC SNe were only observed spectroscopically at lower priority, depending on the availability of telescope time, while other events were initially (photometrically) misidentified as SNe Ia.

The data presented in this paper were collected during three consecutive nights (21-23 August 2008) with the ESO New Technology Telescope (NTT) equipped with EFOSC2. The galaxies observed were those that matched the RA and Dec window best, so this should not introduce any more biases. There is no overlap with the sample of Anderson et al. (2010) and only a small overlap (5 SN hosts) with the sample of Modjaz et al. (2011). The Modjaz et al. (2011) sample also includes SNe that were discovered in "non-targeted" searches (such as the SDSS-II SN Survey) while the Anderson et al. (2010) hosts are mostly "targeted" galaxies.
A list of the observed galaxies is provided in Table 1. This table contains basic information on the host galaxy, the hosted SN, as well as details concerning our observations. We comment further on the critical issue of SN typing in Sect. 2.1. The tabulated offsets are SN galactocentric distances (projected on the plane of the sky), based on the redshift of the host galaxies. The following cosmological parameters have been adopted throughout the paper: $H_{0}=73 \mathrm{~km} \mathrm{~s}^{-1} \mathrm{Mpc}^{-1}, \Omega_{\mathrm{M}}=0.27$, and $\Omega_{\Lambda}=0.73$. For the two galaxies that are not in the Hubble flow (i.e. NGC 1187 and NGC 4981), we used the distances reported by Tully et al. (2009). For the solar metallicity, we adopted the following value: $\log (\mathrm{O} / \mathrm{H})+12=8.69$ (Asplund et al. 2009).

Our observing strategy involved positioning the slit to contain both the explosion site and the galaxy center. This was done at the expense of not observing at parallactic angle, but most objects were observed at low airmasses (Table 1) so any differential slit losses should be minimal. In most cases we used the EFOSC2 grism 11, but other grisms were also used to optimize the resolution in the (restframe) wavelength region 3700-6800 ̊. A $1^{\prime \prime}$ wide slit was used throughout the observing run. It is noted that at the time of observations there was no significant contribution to the measured flux by the underlying SNe. SN 2007kj, the brightest of the SNe discovered in the fall of 2007 in our sample, is not expected to contribute with more than $4 \times 10^{-19} \mathrm{erg} \mathrm{s}^{-1} \mathrm{~cm}^{-2} \AA^{-1}$ in the $V$-band (assuming a late-time evolution similar to the type Ib SN 2007Y; Stritzinger et al. 2009).

All frames were bias-subtracted and flat-fielded with standard tasks in $\mathrm{IRAF}^{1}$. Cosmic rays were removed from the science spectra with the task LACosmic (van Dokkum 2001). The tasks identify, reidentify, and fitcoords were used on the HeAr arcs to create 2D dispersion maps and all 2D spectra were wavelength calibrated with the task transform. We extracted spectra using apall both at the SN location and at the galaxy nucleus. For the SN location we tried to use the smallest aperture possible (e.g. 7-10 pixel wide columns) to minimize contamination from neighboring regions. In some cases this still corresponds to integrated light from areas on the order of $1 \mathrm{kpc}^{2}$, in particular when the effect of seeing is included. This illustrates the limitations of this method, i.e. that the "local" $\mathrm{SN}$ environment probed is in reality a large region, although this method still probes the SN environment better than a nuclear galaxy spectrum. In four cases where no useful signal could be recovered at the $\mathrm{SN}$ location, we resorted to extracting spectra of other regions, which were as nearby as possible to the exact location and which contained sufficient signal. The projected distances on the plane of the sky between the reported regions and the SN locations, at the distance of these galaxies (that all appear face-on), are: $4.63 \mathrm{kpc}$ for MGC+03-43-5, $4.11 \mathrm{kpc}$ for NGC 1187, $7.19 \mathrm{kpc}$ for NGC 214 and $2.42 \mathrm{kpc}$ for NGC 7803. In all cases we first traced the nuclear galaxy spectrum and then used the same trace function for all other apertures.

During our observing run, the Moon was relatively bright and close to many of our targets. The influence of the Moon was manifested as a halo in the blue part of the CCD. This complicated pattern proved difficult to correct for, causing problems in the background removal, especially below $4000 \AA$. Combined with the reduced sensitivity of the CCD in the blue, data below this wavelength therefore have large associated uncertainties.

${ }^{1}$ IRAF is distributed by the National Optical Astronomy Observatory: http://iraf.noao. edu/iraf/web/. 
Table 1. SN host galaxy sample: properties and observing log.

\begin{tabular}{|c|c|c|c|c|c|c|c|c|c|c|c|}
\hline Galaxy & SN & $\begin{array}{c}\mathrm{RA}(\mathrm{J} 2000)^{a} \\
\left({ }^{\circ},{ }^{\prime}{ }^{\prime \prime}\right)\end{array}$ & $\begin{array}{c}\operatorname{Dec}(\mathrm{J} 2000)^{a} \\
\left({ }^{\circ}{ }^{\prime},{ }^{\prime \prime}\right)\end{array}$ & $\begin{array}{c}\text { Offset }^{b} \\
(\mathrm{kpc})\end{array}$ & $M_{\mathrm{B}}{ }^{c}$ & Redshift $^{d}$ & type $e^{e}$ & Sample $^{f}$ & $\begin{array}{l}\text { Resolution } \\
\left(\AA / 1^{\prime \prime}\right)\end{array}$ & $\begin{array}{l}\text { Exposure time } \\
\text { (s) }\end{array}$ & Airmass \\
\hline 2MASXJ21024677-0405233 & $2007 \mathrm{hn}$ & 210246.85 & -040525.2 & 1.06 & -20.35 & 0.0273 & Ic & $\operatorname{CSP}(\mathrm{N})$ & 13.6 & $4 \times 1800$ & 1.14 \\
\hline ESO $153-$ G17 & $2004 \mathrm{ew}$ & 020506.17 & -550631.6 & 5.40 & -20.98 & 0.0218 & $\mathrm{Ib}$ & $\operatorname{CSP}(\mathrm{T})$ & 17.2 & 1800 & 1.13 \\
\hline ESO $552-\mathrm{G} 40$ & $2004 \mathrm{ff}$ & 045846.19 & -213412.0 & 5.47 & -21.05 & 0.0226 & $\mathrm{Ib} / \mathrm{IIb}$ & $\operatorname{CSP}(\mathrm{T})$ & 17.2 & 1800 & 1.11 \\
\hline IC 4837A & 2005aw & 191517.44 & -540824.9 & 6.11 & -21.60 & 0.0094 & Ic & $\operatorname{CSP}(\mathrm{T})$ & $17.2,8.2$ & $2 \times 1200,2 \times 1800$ & $1.13,1.11$ \\
\hline $\mathrm{J} 000109.19+010409.5$ & $2007 \mathrm{nc}$ & 000109.30 & +010406.5 & 8.76 & -19.83 & 0.0860 & $\mathrm{Ib}$ & SDSS $(\mathrm{N})$ & 17.2 & $2 \times 1800$ & 1.44 \\
\hline J001039.34-000310.4 & 2007sj & 001039.63 & -000310.2 & 3.54 & -20.88 & 0.0390 & Ic & SDSS (N) & 17.2 & $2 \times 1800$ & 1.25 \\
\hline J002741.89+011356.6 & $2007 q x$ & 002741.78 & +011359.6 & 5.63 & -20.02 & 0.0800 & Ic & SDSS $(\mathrm{N})$ & 17.2 & $2 \times 1800$ & 1.18 \\
\hline J012314.96-001948.8 & 2006jo & 012314.71 & -001946.7 & 6.79 & -20.59 & 0.0770 & $\mathrm{Ib}$ & SDSS $(\mathrm{N})$ & 17.2 & $2 \times 1800$ & 1.16 \\
\hline $\mathrm{J} 023239.17+003700.1$ & 2006 fo & 023238.89 & +003703.0 & 2.39 & -19.76 & 0.0201 & $\mathrm{Ib}$ & both $(\mathrm{N})$ & 17.2 & $2 \times 1800$ & 1.16 \\
\hline $\mathrm{J} 205121.43+002357.8$ & 2007jy & 205121.43 & +00 2357.8 &..$^{g}$ & -19.61 & 0.1831 & $\mathrm{Ib}$ & SDSS $(\mathrm{N})$ & $16.8,7.0$ & $2100,2 \times$ & $1.45,1.21$ \\
\hline $\mathrm{J} 205519.76+003234.4$ & $2005 \mathrm{hl}$ & 205519.79 & +003234.8 & 5.37 & -19.76 & 0.0230 & $\mathrm{Ib}$ & SDSS $(\mathrm{N})$ & 8.2 & $3 \times 1500$ & 1.18 \\
\hline J213900.63-010138.6 & $2005 \mathrm{hm}$ & 21390.64 & -010138.6 &.$^{g}$ & -14.89 & 0.0347 & $\mathrm{Ib}$ & SDSS $(\mathrm{N})$ & 17.2 & 2100 & 1.30 \\
\hline $\mathrm{J} 223529.00+002856.1$ & $2007 q w$ & 223529.01 & +002856.2 &..$^{g}$ & -19.06 & 0.1507 & Ia & SDSS $(\mathrm{N})$ & $16.8,17.2$ & 1800,1800 & 1.30 \\
\hline KUG $2302+073$ & 2006ir & 230435.68 & +073621.5 & 1.79 & -16.95 & 0.0200 & Ic & $\operatorname{CSP}(\mathrm{N})$ & 17.2 & 695 & 1.41 \\
\hline MGC+03-43-5 & $2005 \mathrm{bj}$ & 164944.74 & +175148.7 & 5.83 & -20.01 & 0.0222 & $\mathrm{IIb}$ & $\operatorname{CSP}(\mathrm{T})$ & 17.2 & $2 \times 1800$ & 1.50 \\
\hline NGC 1187 & $2007 \mathrm{Y}$ & 030235.92 & -225350.1 & 9.83 & -20.17 & 0.0046 & $\mathrm{Ib}$ & $\operatorname{CSP}(\mathrm{T})$ & 17.2 & $2 \times 1800$ & 1.07 \\
\hline NGC 214 & 2006ер & 004124.88 & +252946.7 & 13.44 & -21.62 & 0.0151 & $\mathrm{Ib}$ & $\operatorname{CSP}(\mathrm{T})$ & 17.2 & $2 \times 1800$ & 1.74 \\
\hline NGC 4981 & $2007 \mathrm{C}$ & 130848.80 & -064645.0 & 2.79 & -20.25 & 0.0056 & $\mathrm{Ib} / \mathrm{c}$ & $\operatorname{CSP}(\mathrm{T})$ & 17.2 & $2 \times 1800$ & 2.06 \\
\hline NGC 7364 & $2006 \mathrm{lc}$ & 224424.48 & -000953.5 & 3.14 & -21.21 & 0.0162 & $\mathrm{Ib} / \mathrm{c}$ & both (TN) & 17.2 & 1800 & 1.25 \\
\hline NGC 7803 & $2007 \mathrm{kj}$ & 000119.58 & +130630.6 & 4.15 & -20.88 & 0.0178 & $\mathrm{Ib} / \mathrm{c}$ & $\operatorname{CSP}(\mathrm{T})$ & 17.2. 8.2 & 1200,1800 & 1.42 \\
\hline
\end{tabular}

Notes. The order in the table is alphanumeric. Galaxies that were observed with more than one grism have double entries in the resolution, exposure time and airmass columns. ${ }^{(a)} \mathrm{RA}$ and Dec of the SN. ${ }^{(b)}$ Galactocentric distance of the SN region projected on the plane of the sky (at the distance of the host galaxy). ${ }^{(c)}$ Galaxy absolute magnitude. ${ }^{(d)}$ Drawn from the NASA Extragalactic Database (NED) and SDSS, except for the hosts of SNe 2007hn, 2007nc, 2007jy, 2005hm and 2007qw which have redshifts determined from our spectroscopic observations. ${ }^{(e)}$ See the detailed discussion in Sect. 2.1. ${ }^{(f)}$ Whether the SN belongs to the CSP or the SDSS subsample with the discovery method noted in parentheses: T stands for targeted and $\mathrm{N}$ for non-targeted searches. ${ }^{(g)}$ Either point sources, or the SN location coincides with the galaxy nucleus.

\subsection{SN classifications}

Traditionally, SNe are classified by their optical spectra around maximum light, and the distinction between $\mathrm{SNe} \mathrm{Ib}$ and $\mathrm{SNe} \mathrm{Ic}$ is based on the relative strength of $\mathrm{He}_{\mathrm{I}}$ and $\mathrm{O} \mathrm{I}$ lines (Filippenko 1997; Matheson et al. 2001). The distinction between these subtypes can, however, be a subtle and difficult matter.

Following the temporal evolution of the spectra is usually a great help, especially since the He lines in $\mathrm{SNe} \mathrm{Ib}$ typically become more prominent as time passes, and it is not unusual that $\mathrm{SNe}$ are reclassified as multi-epoch spectra are obtained. In some cases, however, the data coverage and/or quality of the spectrum used to make the classification is poor so a subclassification is difficult, if not impossible. In fact, $\mathrm{SNe} \mathrm{Ib} / \mathrm{c}$ form a continuum, depending on the amount of He that has been stripped off their progenitor. The existence of intermediate objects also consists a challenge for the subclassification scheme.

Great caution should be taken when adopting the reported $\mathrm{SN}$ types, especially when the distinction between types Ib and Ic is important. The spectral classifications that are reported in Table 1 are the result of careful inspection of the spectra that were available to us, and in many cases they differ from what is reported in the IAU or CBET circulars or in the continuously updated Asiago SN catalog (Barbon et al. 1989). The number of reclassifications we had to make is significant (8 out of 20 events). This shows that, if proper care is not taken, even the statistical power of large samples might be affected. The individual cases are briefly discussed below.

For the CSP subsample we note the following differences: SNe 2006ir and 2007hn were discovered and reported as $\mathrm{SNe} \mathrm{Ib} / \mathrm{c}$ by the SN Factory (Pecontal et al. 2006; Buton et al. 2007). Based on multi-epoch CSP spectra, we refine the classication to SNe Ic. SN 2005bj is a SN IIb and not a SN Ic as the strong absorption at $\sim 6350 \AA$ is clearly due to $\mathrm{H} \alpha$ (possibly blended with He 26678 ) and not to Si as initially suspected (Morrell et al. 2005). SNID (Blondin \& Tonry 2007) also shows an excellent overall agreement with other type IIb objects. Similarly, SN 2004ff is a SN Ib (probably even IIb) and not of type Ic, based on prominent He lines detected in a series of 3 spectra. The first CSP spectrum is dated almost 2 weeks after the reported classification spectrum (Modjaz et al. 2004), illustrating the importance of temporal coverage. For the purposes of this paper, $\mathrm{SNe}$ IIb will be grouped together with $\mathrm{SNe}$ Ib. For SNe 2006lc and 2007kj we note a similarity with SN 1999ex (see also Blondin et al. 2006; Olivares et al. 2007), for which an intermediate classification (Ib/c) is often quoted since its He lines are weaker than in prototypical type Ib objects (Hamuy et al. 2002). We have attributed them such a transitional classification, although we also discuss the consequences of including them in the type Ib sample. SN $2007 \mathrm{C}$ is even less of a clear case and for this reason we preferred a mixed Ib/c classification. The CSP SNe Ib/c spectra will be published in Stritzinger et al. (in prep.).

All spectra obtained at the NTT or the NOT for the SDSS-II SN Survey have been carefully reduced and examined again in a comprehensive and systematic way by Östman et al. (2011), and are now publicly available ${ }^{2}$. These include 6 of the $9 \mathrm{SNe} \mathrm{Ib/c}$ from our SDSS subsample. In general, the SDSS-II discovered $\mathrm{SNe}$ have spectra with lower $\mathrm{S} / \mathrm{N}$, suffer significant host galaxy contamination and typically span only $1-2$ epochs, making classification a de facto more difficult task compared to the nearby CSP SNe. An exception is SN 2006fo, for which we have many spectra, including three from the CSP. The examination of this event by Östman et al. (2011) led to the confident revision of its type to Ib (from Ic), which is now also the official SDSS-II classification. We inspected the spectra of the other events and

\footnotetext{
${ }^{2}$ www.physto.se/ linda/spectra/nttnot.html
} 
Table 2. Measured line fluxes and $\mathrm{H} \alpha E W$ at the SN locations.

\begin{tabular}{lccccc}
\hline \hline Galaxy & $\mathrm{H} \beta$ & {$[\mathrm{O}$ III $] \lambda 5008$} & $\mathrm{H} \alpha$ & {$[\mathrm{N}$ II] $\lambda 6585$} & $\mathrm{H} \alpha E W$ \\
\hline 2MASXJ21024677-0405233 & $6.08 \pm 1.60$ & $\ldots$ & $29.64 \pm 1.70$ & $13.32 \pm 1.50$ & $12.8 \pm 0.7$ \\
ESO 153-G17 & $19.74 \pm 4.60$ & $\ldots$ & $49.83 \pm 5.60$ & $20.14 \pm 2.90$ & $20.7 \pm 1.5$ \\
ESO 552-G40 & $\ldots$ & $\ldots$ & $55.65 \pm 7.10$ & $13.27 \pm 4.00$ & $5.6 \pm 0.6$ \\
IC 4837A & $\ldots$ & $\ldots$ & $145.30 \pm 5.60$ & $52.02 \pm 3.90$ & $35.4 \pm 1.7$ \\
J000109.19+010409.5 & $19.21 \pm 3.90$ & $14.48 \pm 4.30$ & $45.20 \pm 6.30$ & $7.40 \pm 4.30$ & $81.7 \pm 11.9$ \\
J001039.34-000310.4 & $21.49 \pm 4.90$ & $\ldots$ & $110.80 \pm 6.60$ & $43.24 \pm 4.70$ & $19.7 \pm 0.8$ \\
J002741.89+011356.6 & $28.22 \pm 6.90$ & $\ldots$ & $121.50 \pm 7.70$ & $26.22 \pm 5.80$ & $14.7 \pm 0.8$ \\
J012314.96-001948.8 & $17.28 \pm 5.30$ & $29.24 \pm 5.70$ & $57.01 \pm 5.90$ & $19.93 \pm 5.60$ & $23.2 \pm 2.2$ \\
J023239.17+003700.1 & $140.80 \pm 11.00$ & $53.45 \pm 13.00$ & $634.80 \pm 14.00$ & $206.70 \pm 10.00$ & $31.7 \pm 0.8$ \\
J205121.43+002357.8 & $125.40 \pm 5.00$ & $170.20 \pm 6.00$ & $317.80 \pm 7.40$ & $56.93 \pm 5.00$ & $49.7 \pm 1.5$ \\
J205519.76+003234.4 & $133.20 \pm 3.80$ & $55.18 \pm 4.30$ & $512.20 \pm 10.80$ & $162.70 \pm 7.80$ & $55.1 \pm 2.4$ \\
J213900.63-010138.6 & $27.83 \pm 4.50$ & $92.26 \pm 4.10$ & $87.23 \pm 6.10$ & $4.07 \pm 5.60$ & $56.2 \pm 4.3$ \\
J223529.00+002856.1 & $165.20 \pm 4.50$ & $542.40 \pm 4.50$ & $431.00 \pm 6.40$ & $32.59 \pm 3.30$ & $146.2 \pm 11.7$ \\
KUG 2302+073 & $49.76 \pm 4.60$ & $87.38 \pm 4.60$ & $185.50 \pm 6.50$ & $29.44 \pm 5.00$ & $69.3 \pm 4.5$ \\
MGC+03-43-5 & $16.75 \pm 2.30$ & $11.42 \pm 8.80$ & $53.71 \pm 4.00$ & $14.82 \pm 2.80$ & $11.8 \pm 0.7$ \\
NGC 1187 & $46.39 \pm 3.40$ & $12.64 \pm 2.80$ & $176.30 \pm 6.50$ & $53.03 \pm 4.40$ & $127.4 \pm 6.7$ \\
NGC 214 & $124.70 \pm 9.60$ & $\ldots$ & $495.50 \pm 14.00$ & $163.20 \pm 8.10$ & $46.1 \pm 1.2$ \\
NGC 4981 & $45.37 \pm 5.30$ & $11.69 \pm 4.80$ & $259.60 \pm 9.30$ & $102.70 \pm 5.30$ & $50.5 \pm 2.1$ \\
NGC 7364 & $7.96 \pm 3.10$ & $\ldots$ & $32.84 \pm 6.60$ & $18.58 \pm 5.90$ & $11.0 \pm 1.3$ \\
NGC 7803 & $280.80 \pm 6.20$ & $51.56 \pm 5.50$ & $1034.00 \pm 8.10$ & $464.00 \pm 5.50$ & $38.7 \pm 0.6$ \\
\hline
\end{tabular}

Notes. The fluxes are given in units of $10^{-17} \mathrm{erg} \mathrm{s}^{-1} \mathrm{~cm}^{-2}$. The $\mathrm{H} \alpha E W$ is in $\AA$. (a) No sufficient signal was recovered at the SN location and the fluxes correspond to a nearby region (see text for the exact distances).

conclude that the most probable types are indeed those that are adopted by SDSS-II, and we therefore retain these classifications. Admittedly, a detailed division between types Ib and Ic SN based on these spectra might be overly ambitious. A more conservative approach is followed by Östman et al. (2011) who prefer a general $\mathrm{SN} \mathrm{Ib/c} \mathrm{classification} \mathrm{for} 2$ events (SNe 2006jo and 2007qx), while for SNe 2007sj and 2007jy, they conclude that even a type $\mathrm{Ib} / \mathrm{c}$ classification is not unambiguous. Finally, there is also the case of SN 2007qw, whose spectral type has been revised from type Ib to type Ia by the SDSS-II collaboration (M. Sako; priv. comm.). We have therefore only included it in our tables and not in the analysis ${ }^{3}$.

\section{Methods and results}

To derive accurate measures of nebular emission lines, which carry information about the gas metallicity and the age of the youngest stellar populations, care must be taken in accounting for the underlying stellar continuum and absorption features. This is particularly relevant for the Balmer lines of young stellar populations. To be able to even recover weak emission lines, we rely on the procedure implemented in the platefit code. This code has been developed and optimized for the analysis of SDSS spectra, as described in Tremonti et al. (2004) and Brinchmann et al. (2004). In summary, the stellar continuum is recovered by fitting stellar population synthesis models to the galaxy spectrum. The template model spectra are simple stellar population (SSP) spectra predicted by the Bruzual \& Charlot (2003) code at ten different ages between 0.005 and $10 \mathrm{Gyr}$ and three metallicities $\left(Z=0.2,1\right.$, and $2.5 Z_{\odot}$, where $\left.Z_{\odot}=0.02\right)$. The templates are first convolved and rebinned to match the spectral resolution and binning of the observed spectra. The code performs a non-negative least-squares fit to the emission-line free

\footnotetext{
3 After the present paper was accepted, new communication revealed that the SDSS-II classification for this SN might be revised again. Modjaz et al. (2011) include it in their broad-lined SN Ic sample. In any case, it is probably better to exclude it due to the related uncertainties.
}

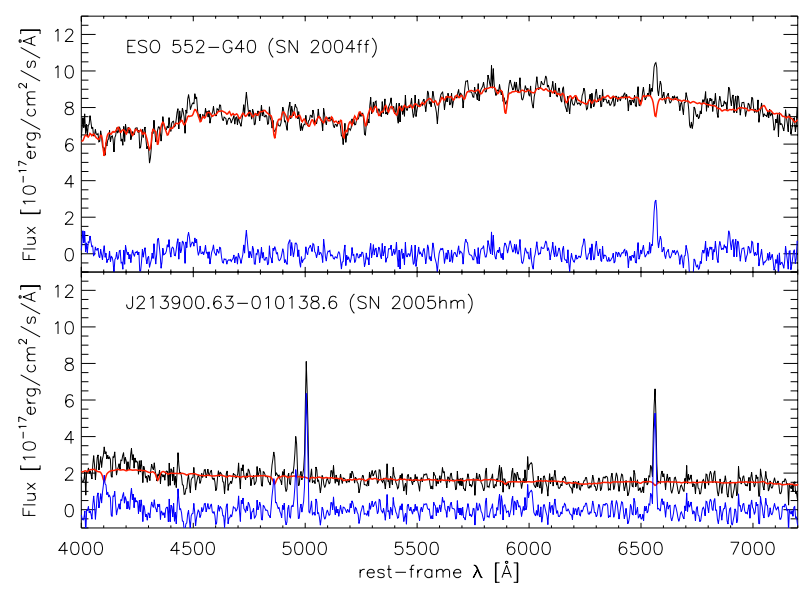

Fig. 1. Examples of stellar continuum fit and extraction of emission line spectrum for two representative cases. The black line shows the original spectrum at the location of the SN explosion. This spectrum is fit with a linear combination of SSP models with dust attenuation as an additional free parameter: the resulting best fit of the stellar continuum is shown in red. By subtracting the best-fit continuum from the original spectrum we obtain the pure emission line spectrum (blue line).

regions of the spectrum finding the best-fitting linear combination of the template spectra with dust attenuation as an additional free parameter. The "pure" emission line galaxy spectrum is recovered by subtracting from the observed spectrum the best-fit continuum and any remaining residuals smoothed on a scale of 200 pixels. Two representative examples are shown in Fig. 1.

We measured emission line fluxes and equivalent widths $(E W)$ from the stellar-continuum-subtracted spectra and tabulated their values in Table 2 . The fluxes were measured by fitting Gaussians with the IRAF task splot. For [N II] $\lambda 6550$, $\mathrm{H} \alpha$, and [ $\left.\mathrm{N}_{\mathrm{II}}\right] \lambda 6585$, we performed de-blending by fitting simultaneously 3 Gaussians with a single FWHM. In Table 2 we have listed the fluxes for $\mathrm{H} \beta$, [O III] $\lambda 5008, \mathrm{H} \alpha$, and [N II] $\lambda 6585$. Some galaxies display many more lines, including [O II] $\lambda 3727$, 
Table 3. Metallicities and age estimates at the local SN environment.

\begin{tabular}{|c|c|c|c|c|c|c|}
\hline Galaxy & SN & type & $\mathrm{O} 3 \mathrm{~N} 2$ & $\mathrm{~N} 2$ & $\begin{array}{c}\text { Age (integr.) } \\
(\mathrm{Gyr})\end{array}$ & $\begin{array}{r}\text { Age }(\min .)^{b} \\
(\mathrm{Myr})\end{array}$ \\
\hline 2MASXJ21024677-0405233 & $2007 \mathrm{hn}$ & Ic & $\ldots$ & $8.70 \pm 0.03$ & $5.00_{-1.57}^{+1.81}$ & $8.6-11.1$ \\
\hline ESO $153-$ G17 & $2004 \mathrm{ew}$ & $\mathrm{Ib}$ & $\ldots$ & $8.68 \pm 0.05$ & $1.56_{-0.63}^{+1.12}$ & $7.3-9.7$ \\
\hline ESO 552-G40 & $2004 \mathrm{ff}$ & $\mathrm{Ib} / \mathrm{IIb}$ & $\ldots$ & $8.55 \pm 0.08$ & $6.89_{-1.49}^{+1.60}$ & $12.3-12.7$ \\
\hline IC $4837 \mathrm{~A}$ & 2005aw & Ic & $\ldots$ & $8.65 \pm 0.02$ & $0.92_{-0.25}^{+0.68}$ & $6.6-6.7$ \\
\hline J000109.19+010409.5 & $2007 \mathrm{nc}$ & $\mathrm{Ib}$ & $8.52 \pm 0.10$ & $8.45 \pm 0.15$ & $0.88_{-0.29}^{+0.67}$ & $6.3-6.8$ \\
\hline J001039.34-000310.4 & $2007 \mathrm{sj}$ & Ic & $\ldots$ & $8.67 \pm 0.03$ & $2.81_{-0.75}^{+1.21}$ & $7.4-9.7$ \\
\hline J002741.89+011356.6 & $2007 \mathrm{qx}$ & Ic & $\ldots$ & $8.52 \pm 0.06$ & $2.04_{-0.59}^{+0.94}$ & $10.4-10.9$ \\
\hline J012314.96-001948.8 & 2006jo & $\mathrm{Ib}$ & $8.51 \pm 0.07$ & $8.64 \pm 0.07$ & $1.89_{-0.76}^{+1.25}$ & $7.1-9.4$ \\
\hline J023239.17+003700.1 & 2006fo & $\mathrm{Ib}$ & $8.71 \pm 0.04$ & $8.62 \pm 0.01$ & $1.79_{-0.64}^{+1.68}$ & 6.7 \\
\hline $\mathrm{J} 205121.43+002357.8$ & 2007jy & $\mathrm{Ib}$ & $8.45 \pm 0.01$ & $8.47 \pm 0.02$ & $3.24_{-2.07}^{+1.04}$ & $7.1-7.4$ \\
\hline $\mathrm{J} 205519.76+003234.4$ & $2005 \mathrm{hl}$ & $\mathrm{Ib}$ & $8.69 \pm 0.01$ & $8.62 \pm 0.01$ & $4.76_{-1.30}^{+1.72}$ & $6.3-6.4$ \\
\hline J213900.63-010138.6 & $2005 \mathrm{hm}$ & $\mathrm{Ib}$ & $\ldots$ & $8.23 \pm 0.20^{c}$ & $4.30_{-2.06}^{+3.04}$ & $6.9-7.2$ \\
\hline $\mathrm{J} 223529.00+002856.1$ & $2007 q w$ & Ia & $8.21 \pm 0.01$ & $8.26 \pm 0.03$ & $2.45_{-0.73}^{+1.22}$ & $5.9-6.1$ \\
\hline KUG $2302+073$ & 2006ir & Ic & $8.40 \pm 0.03$ & $8.44 \pm 0.04$ & $5.25_{-2.27}^{+0.04}$ & $6.6-6.9$ \\
\hline $\mathrm{MGC}+03-43-5$ & $2005 b j$ & IIb & $8.60 \pm 0.11$ & $8.58 \pm 0.05$ & $0.94_{-0.23}^{+0.57}$ & $9.9-11.2$ \\
\hline NGC 1187 & $2007 \mathrm{Y}$ & $\mathrm{Ib}$ & $8.74 \pm 0.03$ & $8.60 \pm 0.02$ & $6.48_{-2.26}^{+2.64}$ & $5.9-6.0$ \\
\hline NGC 214 & 2006ep & $\mathrm{Ib}$ & $\ldots$ & $8.63 \pm 0.01$ & $2.04_{-0.53}^{+0.85}$ & $6.4-6.5$ \\
\hline NGC 4981 & $2007 \mathrm{C}$ & $\mathrm{Ib} / \mathrm{c}$ & $8.79 \pm 0.06$ & $8.67 \pm 0.02$ & $2.40_{-0.68}^{+1.06}$ & 6.4 \\
\hline NGC 7364 & 2006lc & $\mathrm{Ib} / \mathrm{c}$ & $\ldots$ & $8.76 \pm 0.09$ & $3.16_{-1.25}^{+2.14}$ & $9.9-11.7$ \\
\hline NGC 7803 & $2007 \mathrm{kj}$ & $\mathrm{Ib} / \mathrm{c}$ & $8.85 \pm 0.02$ & $8.70 \pm 0.01$ & $4.77_{-1.26}^{+1.55}$ & 6.6 \\
\hline
\end{tabular}

Notes. The tabulated metallicity errors are only the propagated measurement errors. To obtain the total uncertainty in the metallicity one needs to add quadratically a systematic error of 0.14 dex for O3N2 and 0.18 dex for N2 (Pettini \& Pagel 2004). ${ }^{(a)}$ Integrated, luminosity-weighted mean stellar age in the region, obtained by absorption features. ${ }^{(b)}$ Minimum stellar age, obtained by the $\mathrm{H} \alpha E W$ that probes the youngest (most recent) star formation episode. ${ }^{(c)}$ Metallicity computed with the $R_{23}$ method (see text).

[O III] $\lambda 4960$, and [S II] $\lambda \lambda 6717,6731$; however, the tabulated lines are those that are more consistently detected and those used for our metallicity determinations. The fluxes in Table 2 are the ones measured on the emission line spectra (i.e. corrected for stellar absorption). For reference and to facilitate comparisons with the literature, we also provide the fluxes as measured in the original spectra, both at the host galaxy center and at the SN location (Table 4).

\subsection{Metallicity estimates}

To derive metallicities, we used the empirical O3N2 and N2 calibrations described by Pettini \& Pagel (2004). These methods present some advantages over other strong-line diagnostics, by being based on ratios of neighboring lines and are therefore insensitive to extinction and to uncertainties in flux calibration. Given the limited number of [O II] $\lambda 3727$ detections, combined with the large uncertainties in the blue part of the spectra, no advantage would result from using $R_{23}$ or other methods that use this line (see e.g. Kewley \& Dopita 2002). A necessary exception was made in the case of the host of SN 2005hm. For this galaxy, the faintest in our sample, [N II] $\lambda 6585$ was not significantly detected. Assuming a $2 \sigma$ upper limit for the [ $\left.\mathrm{N}_{\text {II }}\right]$ flux yields an upper limit to the (N2) metallicity of $\log (\mathrm{O} / \mathrm{H})+$ $12<8.44$. Since [O II] $\lambda 3727$ is clearly detected for this galaxy and the Balmer decrement is consistent with no host extinction (Osterbrock 1989), we used $R_{23}$. Taking the average between the calibrations of McGaugh (1991) (the lower value) and Zaritsky et al. (1994), we obtain $\log (\mathrm{O} / \mathrm{H})+12=8.23$. To account for the different method used and all the related uncertainties, we assigned a conservative uncertainty of 0.20 dex to this value.

The N2 calibrator presents a $1 \sigma(2 \sigma)$ dispersion of 0.18 (0.41) dex, which is comparable to the dispersion of $R_{23}$-based methods (Pettini \& Pagel 2004). An even smaller dispersion of $0.14(0.25)$ dex can be achieved by using the $\mathrm{O} 3 \mathrm{~N} 2$ method. However, in our case the determination of O3N2 was possible only for some cases, and for this reason we focus on the N2 scale in the rest of the paper. The estimated metallicities for the local SN environments are tabulated in Table 3.

Plotted in Fig. 2 are the metallicities at the SN locations versus the host galaxy luminosity. In addition to our sample, we have also included the $3 \mathrm{SNe}$ Ib that occurred in NGC 2770 for which we conducted the measurements in a similar manner (Thöne et al. 2009). For comparison we also plotted the GRB associated SNe and the broad-lined SNe Ic studied by Modjaz et al. (2008). All metallicities were converted to the N2 scale based on the reported line fluxes (Modjaz et al. 2008; Sollerman et al. 2005; Hammer et al. 2006; Margutti et al. 2007; Wiersema et al. 2007). The righthand panel shows a comparison with the mean N2 values by Anderson et al. (2010) and Modjaz et al. (2011). This is useful to illustrate the corresponding offsets between type Ib and Ic SNe in the three studies.

The SNe Ib ( $N=14$ including the 3 from Thöne et al. 2009) have a mean $\mathrm{N} 2$ metallicity of $8.52 \pm 0.03$ dex. The quoted error is the standard error of the mean, i.e. $\sigma / \sqrt{N}$, where $\sigma$ is the standard deviation. In this case $\sigma=0.13$. The SNe Ic have a mean metallicity of $8.60 \pm 0.05 \mathrm{dex}$ and $\sigma=0.11$ on the same scale. This is, however, based on only $N=5$ events, since many objects 


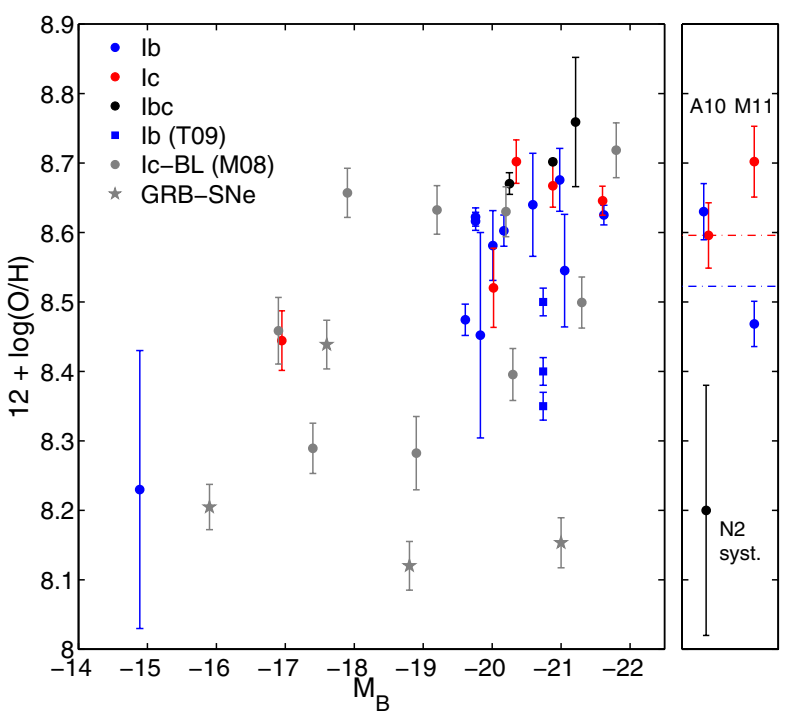

Fig. 2. Metallicities (N2) at the locations of stripped-envelope CC $\mathrm{SNe}$ versus their host galaxy absolute magnitudes. SNe Ib are marked with blue circles (squares for the $3 \mathrm{SNe}$ of Thöne et al. 2009), SNe Ic in red and $\mathrm{SNe} \mathrm{Ib} / \mathrm{c}$ (intermediate or uncertain type) in black. Grey stars are for GRB-SNe and gray circles for SNe Ic-BL (Modjaz et al. 2008). The displayed error bars only include the measurement errors, while all data points have an additional associated uncertainty of 0.18 dex, related to the $1 \sigma$ dispersion in the $\mathrm{N} 2$ index calibration (shown as the black error-bar in the panel to the right). In addition, this panel shows a comparison with the corresponding mean values from Anderson et al. (2010) and Modjaz et al. (2011). Our mean values are marked with dashed-dotted lines.

from our initial sample were reclassified. We note here that these errorbars (like those in the mean values quoted by Anderson et al. 2010; and Modjaz et al. 2011) are underestimated because they do not include the uncertainties in the individual metallicity measurements (largely dominated by the systematic uncertainties in the metallicity calibrators; see Sect. 4.1).

\subsection{Age estimates}

We derive two different estimates for the stellar age at the SN location, namely the luminosity-weighted mean stellar age from the stellar absorption features and the age of the ionizing (youngest) stellar populations from the $\mathrm{H} \alpha E W$. We measure the stellar absorption features of the spectrum subtracted by the Gaussian fits to the nebular emission lines. Estimates of the luminosity-weighted mean age are derived by comparing the observed absorption features with those predicted by a Bruzual \& Charlot (2003)-based model library spanning a comprehensive range in random star formation histories and metallicities, as described in Gallazzi et al. (2005). The constraints are set by a combination of age-sensitive indices $\left(\mathrm{H} \beta\right.$ and $\left.\mathrm{H} \gamma_{\mathrm{A}}+\mathrm{H} \delta_{\mathrm{A}}\right)$ and metal-sensitive indices ([MgFe $]^{\prime}$ and $\left.\left[\mathrm{Mg}_{2} \mathrm{Fe}\right]\right)$ to help break the age-metallicity degeneracy. For each object we derive the probability density function (PDF) of the luminosity-weighted age in this way. We take the median of the PDF as the fiducial age estimate and the 16th and 84th percentiles as the $1 \sigma$ uncertainty range. The results are summarized in Table 3.

One can immediately see that these ages range between 0.8-7 Gyr, i.e. old compared to the lifetimes of massive stars. This is expected since the stellar absorption features probe the mean age integrated over the whole star formation history in the region. In our case, however, it is more relevant to examine the age of the youngest, i.e. latest, star formation episode in this region. The question we want to address is whether there are any SN locations that, despite the large uncertainties, indicate a population that is older than what is allowed by the stellar evolution models for single massive stars. Such an age estimator, which is sensitive to the most recent star formation, is the $\mathrm{H} \alpha E W$ (see e.g. Leitherer et al. 1999; Zackrisson et al. 2001). Even so, the relation between $\mathrm{H} \alpha E W$ and age strongly depends on the star formation history. Unfortunately, our question can only be addressed under the assumption that the latest star formation episode was instantaneous, because if star formation is continuing, massive stars can be born at any time and give rise to CC SNe. This means that a conclusive answer cannot be given, but we believe that any contribution to the "single versus binary" channel discussion deserves effort.

The ages of the young stellar populations in the vicinity of the SNe have been estimated by the measured $\mathrm{H} \alpha E W$ and by comparison with the predictions of Starburst99 (Leitherer et al. 1999, see their Fig. 83) for instantaneous star formation. Our metallicity estimates were used to choose the appropriate table $(Z=0.008$ and $Z=0.02$ are relevant). We were conservative by providing the widest range in ages that are compatible with our measurements. For example, at an $\mathrm{H} \alpha E W$ of $\sim 20 \AA$ at solar metallicity, the models are degenerate, and many ages between 7-10 Myr provide a solution. We therefore provide a range of possible ages (Table 3), rather than one single interpolated age. Furthermore, this range is widened by the uncertainty in our $E W$ measurements and the different possible IMFs examined by Leitherer et al. (1999). Indeed, we see that the ages computed this way are much younger than the luminosity-weighted mean stellar ages estimated from the absorption features and, therefore, represent a lower limit to the age of the $\mathrm{SN}$ regions. These lower limits have been plotted in Fig. 3. We note that Levesque et al. (2010a) used a similar approach and estimated the ages of the young stellar populations in GRB host galaxies from the $\mathrm{H} \beta E W$.

\section{Discussion}

\subsection{Metallicities}

Investigating the metallicities of the $\mathrm{SN}$ environments is important because within the single massive progenitor scenario $\mathrm{SNe}$ Ic are expected to be found in more metal-rich environments than $\mathrm{SNe} \mathrm{Ib}$. This is due to the strong dependence of stellar winds on metallicity (Vink \& de Koter 2005), with stars of the same initial mass suffering more severe mass loss and envelope stripping at higher metallicities.

Our result is intermediate to those of Anderson et al. (2010), who find equal metallicities for SN Ib and Ic environments, and of Modjaz et al. (2011) who find SNe Ic to lie in more metalrich environments than $\mathrm{SNe} \mathrm{Ib}$. Modjaz et al. find a difference of 0.20 dex (in the O3N2 scale) and a high probability for rejecting the null hypothesis that types Ib and Ic explode in similar metallicity environments, based on the Kolmogorov-Smirnov (KS) test $(p$-value $=1 \%)$. In our sample we cannot detect such a significant difference: a KS test provides very weak evidence against such a hypothesis ( $p$-value $=17.1 \%$ ).

We note that the errors quoted until now (i.e. the standard error of the mean), as well as the ones of Anderson et al. and of Modjaz et al., ignore the relatively large errors in the individual metallicity estimates. When the individual errorbars (here $\gtrsim 0.18$ dex) exceed the sample standard deviation $\sigma$ (here $<0.15 \mathrm{dex}$ ), the standard error of the mean underestimates the real uncertainty in the mean value. This is the case here 
Table 4. Measured line fluxes - not corrected for the effect of stellar absorption.

\begin{tabular}{|c|c|c|c|c|c|c|c|c|}
\hline \multirow[t]{2}{*}{ Galaxy } & \multicolumn{2}{|c|}{$\mathrm{H} \beta$} & \multicolumn{2}{|c|}{$\left[\mathrm{O}_{\mathrm{III}}\right] \lambda 5008$} & \multicolumn{2}{|c|}{$\mathrm{H} \alpha$} & \multicolumn{2}{|c|}{$[\mathrm{N}$ II] $\lambda 6585$} \\
\hline & central & local & central & local & central & local & central & local \\
\hline 2MASXJ21024677-0405233 & & $\ldots$ & $\ldots$ & $\ldots$ & $148.50 \pm 4.30$ & $22.68 \pm 1.90$ & $67.90 \pm 3.70$ & $11.63 \pm 1.50$ \\
\hline ESO $153-$ G17 & $\ldots$ & $\ldots$ & $\ldots$ & $\ldots$ & $898.90 \pm 15.00$ & $43.37 \pm 4.40$ & $537.00 \pm 16.00$ & $19.13 \pm 3.10$ \\
\hline ESO 552-G40 & $\ldots$ & $\ldots$ & $\ldots$ & $\ldots$ & $\ldots$ & $36.75 \pm 5.20$ & $144.10 \pm 17.00$ & $12.27 \pm 4.40$ \\
\hline IC $4837 \mathrm{~A}$ & $\ldots$ & $4.55 \pm 1.90$ & $\ldots$ & $9.26 \pm 5.20$ & $\ldots$ & $125.70 \pm 2.20$ & $445.70 \pm 12.00$ & $47.10 \pm 2.10$ \\
\hline J000109.19+010409.5 & $44.22 \pm 7.70$ & $\ldots$ & $39.81 \pm 7.10$ & $\ldots$ & $524.90 \pm 12.00$ & $34.39 \pm 5.00$ & $190.10 \pm 9.30$ & $4.90 \pm 3.60$ \\
\hline J001039.34-000310.4 & $\ldots$ & $\ldots$ & $\ldots$ & $\ldots$ & $450.40 \pm 12.00$ & $94.81 \pm 4.30$ & $177.00 \pm 8.90$ & $35.34 \pm 4.20$ \\
\hline J002741.89+011356.6 & $\ldots$ & $\ldots$ & $\ldots$ & $\ldots$ & $155.40 \pm 10.00$ & $85.59 \pm 7.60$ & $59.48 \pm 7.80$ & $22.08 \pm 5.50$ \\
\hline J012314.96-001948.8 & $26.34 \pm 11.00$ & $\ldots$ & $131.90 \pm 12.00$ & $20.21 \pm 7.20$ & $621.00 \pm 16.00$ & $27.49 \pm 4.60$ & $294.30 \pm 12.00$ & $15.14 \pm 3.60$ \\
\hline $\mathrm{J} 023239.17+003700.1$ & $56.45 \pm 13.00$ & $41.49 \pm 11.00$ & $89.82 \pm 16.00$ & $40.42 \pm 12.00$ & $960.10 \pm 19.00$ & $531.30 \pm 13.00$ & $422.30 \pm 13.00$ & $185.10 \pm 8.70$ \\
\hline $\mathrm{J} 205121.43+002357.8$ & $55.74 \pm 3.90$ & $55.74 \pm 3.90$ & $133.20 \pm 5.30$ & $133.20 \pm 5.30$ & $228.80 \pm 6.80$ & $228.80 \pm 6.80$ & $42.19 \pm 4.00$ & $42.19 \pm 4.00$ \\
\hline $\mathrm{J} 205519.76+003234.4$ & $95.43 \pm 7.10$ & $93.31 \pm 2.40$ & $44.62 \pm 12.00$ & $45.62 \pm 2.80$ & $1270.00 \pm 15.00$ & $477.20 \pm 5.40$ & $407.80 \pm 11.00$ & $162.30 \pm 3.90$ \\
\hline $\mathrm{J} 213900.63-010138.6$ & $19.05 \pm 3.10$ & $19.05 \pm 3.10$ & $82.52 \pm 3.30$ & $82.52 \pm 3.30$ & $78.23 \pm 4.30$ & $78.23 \pm 4.30$ & $4.96 \pm 6.10$ & $4.96 \pm 6.10$ \\
\hline $\mathrm{J} 223529.00+002856.1$ & $127.10 \pm 3.30$ & $127.10 \pm 3.30$ & $448.80 \pm 3.10$ & $448.80 \pm 3.10$ & $390.00 \pm 4.80$ & $390.00 \pm 4.80$ & $29.00 \pm 3.00$ & $29.00 \pm 3.00$ \\
\hline KUG $2302+073$ & $\ldots$ & $35.03 \pm 4.20$ & $\ldots$ & $79.24 \pm 3.80$ & $\cdots$ & $162.80 \pm 5.00$ & $\ldots$ & $26.23 \pm 3.30$ \\
\hline $\mathrm{MGC}+03-43-5$ & $\ldots$ & $\ldots$ & $38.63 \pm 7.80$ & $\ldots$ & $432.70 \pm 9.10$ & $41.03 \pm 2.70$ & $148.00 \pm 6.40$ & $13.91 \pm 2.40$ \\
\hline NGC 1187 & $3557.00 \pm 34.00$ & $41.92 \pm 3.40$ & $450.20 \pm 20.00$ & $12.95 \pm 2.90$ & $25750.00 \pm 30.00$ & $172.60 \pm 6.50$ & $12200.00 \pm 24.00$ & $51.36 \pm 3.60$ \\
\hline NGC 214 & $\ldots$ & $75.65 \pm 7.50$ & $1066.00 \pm 26.00$ & $\ldots$ & $507.00 \pm 43.00$ & $436.00 \pm 14.00$ & $1528.00 \pm 45.00$ & $146.10 \pm 8.80$ \\
\hline NGC 4981 & $\ldots$ & $24.82 \pm 5.50$ & $\ldots$ & $\ldots$ & $2827.00 \pm 29.00$ & $215.30 \pm 7.50$ & $1665.00 \pm 23.00$ & $88.03 \pm 6.30$ \\
\hline NGC 7364 & $\ldots$ & $\ldots$ & $\ldots$ & $\ldots$ & $1155.00 \pm 29.00$ & $26.34 \pm 3.20$ & $718.20 \pm 19.00$ & $17.61 \pm 3.20$ \\
\hline NGC 7803 & $\ldots$ & $158.90 \pm 4.00$ & $\ldots$ & $36.65 \pm 3.20$ & $3450.00 \pm 18.00$ & $786.80 \pm 5.90$ & $2250.00 \pm 17.00$ & $365.30 \pm 3.70$ \\
\hline
\end{tabular}

Notes. The fluxes are given in units of $10^{-17} \mathrm{erg} \mathrm{s}^{-1} \mathrm{~cm}^{-2}$ both for the galaxy central regions and for the local SN environments.

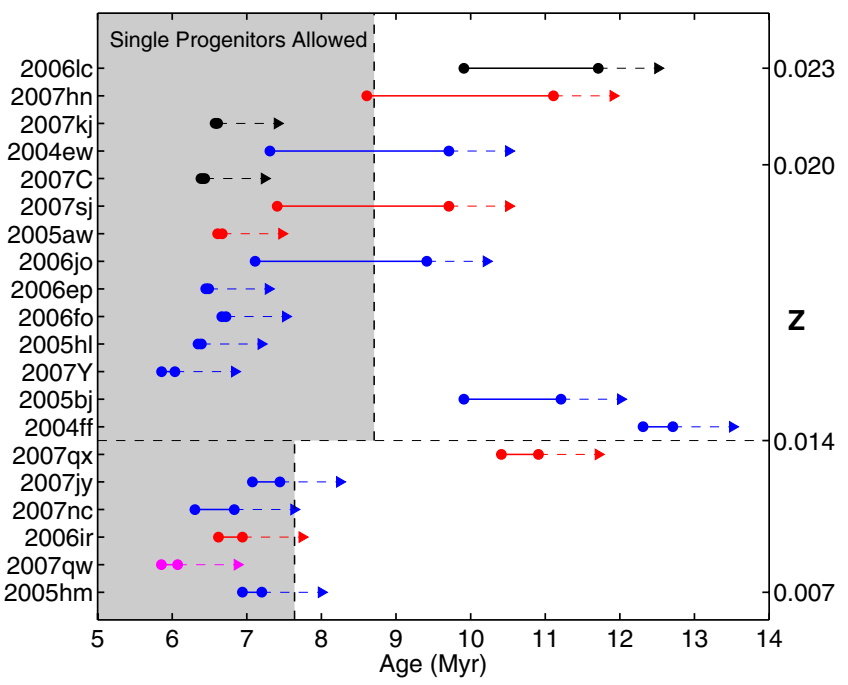

Fig. 3. Ages of the youngest stellar populations at the SN locations, estimated through the measured $\mathrm{H} \alpha E W$ and Starburst99 (Leitherer et al. 1999) (range denoted by solid line; see text). These set a lower limit (dashed line) to the age of the SN birthplace. The SN environments were ordered by ascending metallicity, and some key values are indicated on the righthand axis, but this is only for illustration purposes as this axis does not have any meaningful scale. The color coding is the same as in Fig. 2: blue for $\mathrm{SNe} \mathrm{Ib}$, red for $\mathrm{SNe}$ Ic and black for intermediate $\mathrm{SNe} \mathrm{Ib} / \mathrm{c}$. The interloper type Ia SN 2007qw is in magenta. The horizontal dashed line separates the galaxies into two metallicity groups depending on whether a comparison with $Z=0.008$ or $Z=0.02$ models is more appropriate. The vertical dashed lines denote the predictions of the Geneva evolutionary codes for the lifetimes of single massive stars of $25 M_{\odot}$ at $Z=0.02$ and $30 M_{\odot}$ at $Z=0.008$ (Meynet \& Maeder $2003,2005)$. The allowed ages for single progenitors of $\mathrm{SNe} \mathrm{Ib} / \mathrm{c}$ are illustrated by the gray-shaded area.

because the individual metallicity errors are dominated by the uncertainty in the N2 (or O3N2) calibration. In that case, an error propagation of the individual errors in the calculation of the mean is more appropriate. The same results can be obtained by a simple Monte Carlo (MC) simulation where the individual metallicities are perturbed around their mean value (and within the provided errors) and a metallicity mean is computed in each realization. Such a calculation reveals that the true uncertainties in the mean metallicities are $8.52 \pm 0.05$ for $\mathrm{SNe} \mathrm{Ib}$ and $8.60 \pm 0.08$ for SNe Ic. In the rest of the paper all quoted errors are computed with this method. In addition, by performing a $\mathrm{KS}$ test for each MC realization, we find that quoting a single $p$-value is simplistic but that there is a distribution of generated $p$-values with an associated confidence interval (here $0.007<p<0.483$ at the $68 \%$ confidence level). We therefore find no significant evidence that types Ib and Ic SNe explode in different environments. We believe that taking this into account would also lower the significance of the 0.20 dex difference reported by Modjaz et al. (2011).

It is not obvious why the results from the three samples differ slightly, especially for the SN Ib environments. Unlike the other studies, we have measured fluxes in spectra where the stellar continuum has been subtracted (Sect. 3), but this is not what causes the difference: if we use the original spectra (Table 4) we obtain N2 $=8.54 \pm 0.05 \mathrm{dex}$ for SNe Ib and $8.61 \pm 0.08 \mathrm{dex}$ for SNe Ic. The N2 metallicities are on average a little lower in the stellar-continuum-subtracted spectra (as the corrected $\mathrm{H} \alpha$ emission is enhanced), but the offset between types Ib and Ic in our sample remains at the same level. We have compared our results to those that can be obtained on SN spectra that were available to us, a measurement of metallicity based on lines from the host galaxy was possible (11 cases), and we have found very good agreement. Overall good agreement was also obtained for the nuclear metallicities of the five galaxies in our sample that possess an SDSS spectrum. At the time this work was accepted for publication, the version of Modjaz et al. (2011) did not provide individual line fluxes or metallicities, and it was thus not possible to make direct comparisons for the five host galaxies that are common to our samples ${ }^{4}$. Anderson et al. (2010) provide

4 This information is available in the final version of their paper. At this stage, however, it was not possible to include a detailed comparison. 
metallicities for the individual hosts on the $\mathrm{N} 2$ and $\mathrm{O} 3 \mathrm{~N} 2$ scales, but there is no overlap between our samples. By merging the two samples (using our fluxes in Table 4 to avoid differences in methodology), we obtained $8.58 \pm 0.04$ dex for $\mathrm{SNe} \mathrm{Ib}(N=24)$ and $8.60 \pm 0.05$ dex for SNe Ic $(N=19)$, i.e. a result intermediate between the two (and qualitatively very similar to Anderson et al. 2010). This, of course, was expected, but we argue below that, at present, a merging of the samples is perhaps not very meaningful.

The Anderson et al. (2010) sample has SN classifications drawn from the Asiago SN catalog, and we have demonstrated in Sect. 2.1 that this can be risky. Furthermore, the Anderson et al. (2010) sample is likely to be biased towards higher metallicities because it studies $\mathrm{SNe}$ that have been discovered in targeted searches of bright nearby galaxies. Within our own sample, all CC SNe discovered by targeted searches $(N=9)$ have a mean metallicity of $8.65 \pm 0.06 \mathrm{dex}$, while non targeted searches $(N=11)$ give $8.56 \pm 0.06 \mathrm{dex}(\mathrm{SN} 2006 \mathrm{c}$ is included in both samples). In addition, the former seem to cluster more tightly around their mean (close to solar) value $(\sigma=0.07)$, while the latter present a much larger dispersion $(\sigma=0.15)$. This difference is at least as strong as the one found between types Ib and Ic SNe, and it stems from the galaxy mass-metallicity relation (Tremonti et al. 2004). Also, this is additional evidence that the method of $\mathrm{SN}$ discovery is a constraining factor in the observed environmental properties of SNe. It is possible that part of the reason Anderson et al. (2010) find relatively higher metallicities is that they explored a smaller region of the mass-metallicity parameter space. In fact, evidence is mounting that even SN demographics might differ when different types of host galaxies are probed by different types of searches (e.g. Arcavi et al. 2010). We should therefore be careful before comparing two dissimilar samples.

Concerning SNe Ic-BL $(N=9)$, on the N2 scale they have $8.51 \pm 0.06$ dex $(\sigma=0.16)$ and we thus confirm that their environments are closer to the $\mathrm{SN} \mathrm{Ib}$ distribution than to the normal SN Ic distribution (Modjaz et al. 2011; Arcavi et al. 2010). Finally GRB-SNe, as pointed out by Modjaz et al. (2008), are found in lower metallicity environments $(8.23 \pm 0.09$ dex; $\sigma=0.14$ on this scale). The latest nearby event of this kind (GRB 100316D/SN 2010bh) also seems to follow this picture (Chornock et al. 2011; Starling et al. 2011). As a matter of fact, low metallicity has been proposed to be necessary for the formation of a GRB jet (Yoon \& Langer 2005; Woosley \& Heger 2006) in an event that would otherwise appear as a regular stripped $\mathrm{CC}$ explosion (such as the ones studied in this paper). Today, however, GRBs have been discovered in high-metallicity environments (Levesque et al. 2010b; Hashimoto et al. 2010), while the fact that we usually detect them at low metallicities can be attributed to a selection bias, such as dust (e.g. Fynbo et al. 2009) or star formation rate (Kocevski \& West 2011; Mannucci et al. 2011).

We mentioned earlier that for four galaxies, the measurements were made at distances of $2-7 \mathrm{kpc}$ from the $\mathrm{SN}$ (and closer to the galaxy center). Metallicity gradients (e.g. Zaritsky et al. 1994; van Zee et al. 1998) make it possible that the metallicities at the actual SN sites are lower. By using the equation derived by Boissier \& Prantzos (2009), we estimate that differences ranging between $0.08-0.14$ dex should be subtracted by the tabulated values to obtain the SN location metallicity for these four galaxies. Since three of them are of type Ib (the fourth classified as $\mathrm{Ib} / \mathrm{c}$ ), this will have an implication for the sample mean value, lowering it a little. Indeed, assuming these values are correct, the offset between the types Ic and Ib metallicity increases to $0.10 \mathrm{dex}$, becoming slightly more significant.
We caution, however, that these authors note that metallicity gradients might be flatter than the ones they use (e.g. Bresolin et al. 2004) and that this average approach is more suitable for larger statistical samples than for individual galaxies. Indeed, for MGC+03-43-5 we did not detect any significant metallicity gradient from the nucleus to the location where the spectrum was extracted (half way to the $\mathrm{SN}$ location).

There is also the question of SN classifications and how this can affect our samples. For instance, if SNe 2006lc and 2007kj are included in the $\mathrm{SN}$ Ib distribution, rather than in the intermediate type $\mathrm{Ib} / \mathrm{c}$ sample, then our estimate for the mean $\mathrm{SN} \mathrm{Ib}$ metallicity increases by $0.03 \mathrm{dex}$. We propose that in the future, as more and better host galaxy and SN data become available, such studies should focus on the metallicity dependence of a continuous SN property, such as the strength of the He lines, rather than resorting to a bold grouping of either type Ib or type Ic SNe. Such a methodology is, however, beyond the scope of the present paper.

Summarizing, based on the present sample and a proper evaluation of the errors, we can identify a tentative trend (0.08 dex for our fiducial case) towards, on average, finding SNe Ic in more metal-rich environments than $\mathrm{SNe} \mathrm{Ib}$, but this is not a significant result. Given that there is no precise model that quantifies the predicted difference in the mean environmental metallicities between types Ib and Ic SNe, it is difficult to assess the value of this trend. Such a quantitative prediction is difficult because the effect of metallicity has to be disentangled from the one of the progenitor initial mass, which also has an observable effect on the types of CC SNe (Anderson \& James 2008; Kelly et al. 2008), and the exact masses of our SNe are not known. In addition, recent models show that metallicity-driven winds can also play a role in binary evolution at least for some mass ranges (Yoon et al. 2010). Overall, however, the observed trend (if true) does not qualitatively disfavor a single progenitor origin for $\mathrm{SNe} \mathrm{Ib} / \mathrm{c}$.

\subsection{Ages}

The (minimum) ages of the regions that were derived in Sect. 3.2 (with the $\mathrm{H} \alpha E W$ method) are all relatively young in an astrophysical context. They range from 6-13 Myr, in broad agreement with the expectation that they should contain massive stars.

It is possible to make a more quantitative comparison with the lifetimes of single massive stars that explode as $\mathrm{SNe} \mathrm{Ib/c} \mathrm{as}$ predicted by the Geneva evolutionary models (Meynet $\&$ Maeder 2003, 2005; Georgy et al. 2009). We again used two reference cases, $Z=0.008$ and $Z=0.02$, and the horizontal dashed line in Fig. 3 separates our SN environments into two groups, depending on which metallicity is more relevant for them. According to Georgy et al. (2009), the lower mass limit above which stars explode as $\mathrm{SNe} \mathrm{Ib} / \mathrm{c}$ at $Z=0.008$ is $30 M_{\odot}$, while this is lowered to $25 M_{\odot}$ at $Z=0.02$ due to the increased effect of wind mass loss. The expected lifetimes of stars of these masses and metallicities are 7.6 and 8.7 Myr, respectively ${ }^{5}$ (Meynet \& Maeder 2003, 2005). These ages represent upper limits of the lifetimes of stars that explode as $\mathrm{SNe} \mathrm{Ib/c} \mathrm{because} \mathrm{more} \mathrm{massive} \mathrm{stars} \mathrm{will}$ explode even sooner. These limits are indicated with the vertical dashed lines in Fig. 3. We thus expect all $\mathrm{SNe} \mathrm{Ib} / \mathrm{c}$ that come from the evolution of single massive stars to be found to the left of these lines (in the gray shaded area). On the contrary, any $\mathrm{SN}$ that exploded outside this area could have a longer progenitor lifetime and may be due to binary evolution (without this

5 These are the lifetimes at the end of core He burning. The remaining lifetimes until core-collapse are however insignificant. 
possibility being excluded for the $\mathrm{SNe}$ in the gray area). Indeed, WR stars are predicted to occur over a wider range of ages in stellar populations that include binaries (Eldridge \& Stanway 2009). We note that despite progress in modeling the evolution of binary stars (e.g. Fryer et al. 2007; Eldridge et al. 2008; Yoon et al. 2010), it is not straightforward to deduce typical lifetimes for systems leading to $\mathrm{SNe} \mathrm{Ib} / \mathrm{c}$ to make a direct comparison with our data. The typical timescales depend on a series of parameters and are not as constrained as for single evolutionary models, ranging from very young (similar to single stars) to very old.

We observe that the lower limits for the ages of most SN host regions are compatible with the single progenitor scenario. There are also, however, a few that seem to contradict it. These are SN 2006lc in NGC 7364, SN 2005bj in MGC+03-43-5, and most notably, SN 2004ff in ESO 552-G40 and SN 2007qx in J002741.89+011356.6. In addition, the problem is more severe in the case of the He-deficient SNe Ic that originate from even more massive progenitors ( $39 M_{\odot}$ at $Z_{\odot}$; Georgy et al. 2009). The lifetimes of these stars are even shorter $\left(<5 \mathrm{Myr}\right.$ at $Z_{\odot}$; Meynet $\&$ Maeder 2003), which would also create a problem for the type Ic SNe 2007sj, 2005aw and, especially, 2007hn. This raises the number of potential discrepancies to 7 out of 19 cases.

As already discussed, these comparisons have been made against the SN region age lower limits. The areas probed by the slit, however, also contain older stellar populations as is clearly indicated by the higher luminosity-weighted mean stellar ages. In principle, more $\mathrm{SNe}$ can originate in regions that are older than the limits in Fig. 3 and there could be more explosions attributed to binarity than in this limiting case. In addition, these stellar lifetimes were for the Geneva rotating models. Nonrotating models typically give lifetimes $\sim 15 \%$ shorter for the same stars (Meynet \& Maeder 2003, 2005). Restricting the comparison to the Geneva models is not a decisive factor since other models give similar (or higher) ZAMS mass limits leading to $\mathrm{SNe} \mathrm{Ib} / \mathrm{c}$ (34 $M_{\odot}$; Heger et al. 2003) and similar (or shorter) timescales for their evolution (e.g. Woosley et al. 1993; Limongi \& Chieffi 2003).

On the other hand, it is true that the quoted discrepancies are small on an absolute scale (between 1-4 Myr) and that star formation is present in almost all SN regions we examined (with the exception of the $4 \mathrm{SNe}$ where no significant flux was recovered at the exact location to allow us measure an $E W$ ). That is, we did not find any clear case indicating a very old environment (as e.g. Perets et al. 2011, for SN 2005cz), which would unambiguously demand a binary channel explosion (always keeping in mind these four galaxies). The most serious concern, however, is that this exercise was made under the assumption of an instantaneous star formation episode. If the SN birthplace is still forming stars, no such strict limits can be placed through the $\mathrm{H} \alpha$ emission. We have no means of assessing the validity of such an assumption (which is indeed challenged by the presence of older stellar generations as indicated by the relatively old mean stellar ages). For this reason our conclusions are weak: we cannot unambiguously claim that the above-mentioned SNe had binary progenitors, but we can propose them as good candidates and we suggest that this possibility is also investigated by other means (e.g. through the SN properties). It is intriguing to point out that two of these explosions are of type IIb, i.e. similar to the prototype SN 1993J, the explosion for which the strongest evidence for binarity exists (e.g. Podsiadlowski et al. 1993; Nomoto et al. 1993; Maund et al. 2004).

As a parenthesis, that the lower limit derived for the thermonuclear SN 2007qw environment is as low as $6 \mathrm{Myr}$ is no surprise and should not raise any concerns. In contrast to CC SNe, this lower limit does not constrain the age of the $\mathrm{SN}$ progenitor in a meaningful way, because it is not comparable to the lifetimes of the suspected progenitor systems. That SNe Ia are often associated with young stellar populations $(\sim 50-100 \mathrm{Myr})$ is well known (e.g. Mannucci et al. 2006).

\section{Conclusions}

We have compiled a sample of 20 well-observed stripped CC SNe. Particular attention was paid to classifying each object, and in many cases our spectral typing differs from what has been reported previously in the literature. We obtained spectra of the host galaxies for these $\mathrm{SNe}$ at the exact $\mathrm{SN}$ location using the NTT (+EFOSC2) and fit these spectra with SSP templates in order to estimate and subtract the stellar continuum contribution.

The local metallicities were computed on the N2 scale and found to be, after a proper treatment of the systematic errors, $8.52 \pm 0.05$ dex for $\mathrm{SNe} \mathrm{Ib}$ and $8.60 \pm 0.08$ dex for SNe Ic. This may indicate a trend toward increasing metallicity from $\mathrm{SNe} \mathrm{Ib}$ to Ic, as expected by single evolutionary models, but it is not a statistically significant result. A comparison with other studies in the literature was made and differences were discussed.

The ages of the SN environs were estimated by comparing absorption features with population synthesis models (giving a luminosity-weighted mean stellar age) and by the measured $\mathrm{H} \alpha E W$. The latter method probes the ionizing youngest stellar populations and was thus used to place lower limits on the ages of the SN birthplaces. These lower limits were compared with upper limits on the lifetimes of single massive stars as computed by the Geneva evolutionary models. For a number of $\mathrm{SNe}(7 / 19)$, these limits were found to be mutually incompatible, possibly indicating that they resulted from binary evolution. The discrepancies were, however, small (between 1-4 Myr), and all the $\mathrm{SN}$ regions we examined included areas of recent $(<15 \mathrm{Myr})$ star formation. In addition, this conclusion was subject to the assumption that the $\mathrm{SN}$ progenitor was born during an instantaneous star formation episode.

Based on these results, we are not able to conclusively rule out any of the evolutionary paths leading to stripped SN explosions. The single progenitor channel seems consistent overall with the observations, while binary evolution might have been more likely for a few explosions in our sample, due to their associated ages. We speculate, in line with many other authors, that stripped SNe probably result from more than one channel. Detailed studies of the individual events and their environmnents are needed in order to reveal the nature of each one separately.

Acknowledgements. We acknowledge helpful comments by Justyn Maund, Christina Thöne, Erik Zackrisson, and the referee, Phil James. We thank Linda Östman and Masao Sako for discussions regarding the SDSS-II SN classifications. We are grateful to Christy Tremonti and Jarle Brinchmann for making their platefit code available to us. G.L. is supported by a grant from the Carlsberg foundation. M.J.M. acknowledges the support of the UK Science \& Technology Facilities Council. The Dark Cosmology Centre is funded by the Danish National Research Foundation.

\section{References}

Anderson, J. P., \& James, P. A. 2008, MNRAS, 390, 1527

Anderson, J. P., \& James, P. A. 2009, MNRAS, 399, 559

Anderson, J. P., Covarrubias, R. A., James, P. A., Hamuy, M., \& Habergham, S. M. 2010, MNRAS, 1175

Arcavi, I., Gal-Yam, A., Kasliwal, M. M., et al. 2010, ApJ, 721, 777

Asplund, M., Grevesse, N., Sauval, A. J., \& Scott, P. 2009, ARA\&A, 47, 481

Barbon, R., Cappellaro, E., \& Turatto, M. 1989, A\&AS, 81, 421

Blondin, S., \& Tonry, J. L. 2007, ApJ, 666, 1024 
Blondin, S., Modjaz, M., Kirshner, R., Challis, P., \& Calkins, M. 2006, Central Bureau Electronic Telegrams, 699, 1

Boissier, S., \& Prantzos, N. 2009, A\&A, 503, 137

Bresolin, F., Garnett, D. R., \& Kennicutt, Jr., R. C. 2004, ApJ, 615, 228

Brinchmann, J., Charlot, S., White, S. D. M., et al. 2004, MNRAS, 351, 1151

Bruzual, G., \& Charlot, S. 2003, MNRAS, 344, 1000

Buton, C., Copin, Y., Gangler, E., et al. 2007, Central Bureau Electronic Telegrams, 1050, 1

Chornock, R., Berger, E., Levesque, E. M., et al. 2011, ApJL, submitted [arXiv: 1004.2262]

Crowther, P. A. 2007, ARA\&A, 45, 177

Eldridge, J. J., \& Stanway, E. R. 2009, MNRAS, 400, 1019

Eldridge, J. J., Izzard, R. G., \& Tout, C. A. 2008, MNRAS, 384, 1109

Filippenko, A. V. 1997, ARA\&A, 35, 309

Frieman, J. A., Bassett, B., Becker, A., et al. 2008, AJ, 135, 338

Fryer, C. L., Mazzali, P. A., Prochaska, J., et al. 2007, PASP, 119, 1211

Fynbo, J. P. U., Jakobsson, P., Prochaska, J. X., et al. 2009, ApJS, 185, 526

Gallazzi, A., Charlot, S., Brinchmann, J., White, S. D. M., \& Tremonti, C. A. 2005, MNRAS, 362, 41

Georgy, C., Meynet, G., Walder, R., Folini, D., \& Maeder, A. 2009, A\&A, 502, 611

Hammer, F., Flores, H., Schaerer, D., et al. 2006, A\&A, 454, 103

Hamuy, M., Maza, J., Pinto, P. A., et al. 2002, AJ, 124, 417

Hamuy, M., Folatelli, G., Morrell, N. I., et al. 2006, PASP, 118, 2

Hashimoto, T., Ohta, K., Aoki, K., et al. 2010, ApJ, 719, 378

Heger, A., Fryer, C. L., Woosley, S. E., Langer, N., \& Hartmann, D. H. 2003, ApJ, 591, 288

Kelly, P. L., Kirshner, R. P., \& Pahre, M. 2008, ApJ, 687, 1201

Kessler, R., Becker, A. C., Cinabro, D., et al. 2009, ApJS, 185, 32

Kewley, L. J., \& Dopita, M. A. 2002, ApJS, 142, 35

Kocevski, D., \& West, A. A. 2011, ApJL, submitted [arXiv: 1011.4060]

Leitherer, C., Schaerer, D., Goldader, J. D., et al. 1999, ApJS, 123, 3

Leloudas, G., Sollerman, J., Levan, A. J., et al. 2010, A\&A, 518, A29

Levesque, E. M., Berger, E., Kewley, L. J., \& Bagley, M. M. 2010a, AJ, 139, 694

Levesque, E. M., Kewley, L. J., Graham, J. F., \& Fruchter, A. S. 2010b, ApJ, 712, L26

Limongi, M., \& Chieffi, A. 2003, ApJ, 592, 404

Mannucci, F., Della Valle, M., \& Panagia, N. 2006, MNRAS, 370, 773

Mannucci, F., Salvaterra, R., \& Campisi, M. A. 2011, MNRAS, 439

Margutti, R., Chincarini, G., Covino, S., et al. 2007, A\&A, 474, 815

Matheson, T., Filippenko, A. V., Li, W., Leonard, D. C., \& Shields, J. C. 2001 AJ, 121,1648
Maund, J. R., Smartt, S. J., Kudritzki, R. P., Podsiadlowski, P., \& Gilmore, G. F. 2004, Nature, 427, 129

McGaugh, S. S. 1991, ApJ, 380, 140

Meynet, G., \& Maeder, A. 2003, A\&A, 404, 975

Meynet, G., \& Maeder, A. 2005, A\&A, 429, 581

Modjaz, M., Challis, P., Kirshner, R., et al. 2004, IAU Circ., 8428, 3

Modjaz, M., Kewley, L., Kirshner, R. P., et al. 2008, AJ, 135, 1136

Modjaz, M., Kewley, L., Bloom, J. S., et al. 2011, ApJ, 731, L4

Morrell, N., Hamuy, M., Folatelli, G., \& Contreras, C. 2005, Central Bureau Electronic Telegrams, 138, 1

Nomoto, K., Suzuki, T., Shigeyama, T., et al. 1993, Nature, 364, 507

Olivares, F., Folatelli, G., \& Morrell, N. 2007, Central Bureau Electronic Telegrams, 1093, 1

Osterbrock, D. E. 1989, Astrophysics of gaseous nebulae and active galactic nuclei, ed. D. E. Osterbrock

Östman, L., Nordin, J., Goobar, A., et al. 2011, A\&A, 526, A28

Pecontal, E., Rigaudier, G., Antilogus, P., et al. 2006, Central Bureau Electronic Telegrams, 658, 1

Perets, H. B., Gal-yam, A., Crockett, R. M., et al. 2011, ApJ, 728, L36

Pettini, M., \& Pagel, B. E. J. 2004, MNRAS, 348, L59

Podsiadlowski, P., Joss, P. C., \& Hsu, J. J. L. 1992, ApJ, 391, 246

Podsiadlowski, P., Hsu, J. J. L., Joss, P. C., \& Ross, R. R. 1993, Nature, 364, 509

Prantzos, N., \& Boissier, S. 2003, A\&A, 406, 259

Prieto, J. L., Stanek, K. Z., \& Beacom, J. F. 2008, ApJ, 673, 999

Smartt, S. J. 2009, ARA\&A, 47, 63

Smith, N., Li, W., Filippenko, A. V., \& Chornock, R. 2011, MNRAS, 412, 1522

Sollerman, J., Östlin, G., Fynbo, J. P. U., et al. 2005, New Astron., 11, 103

Starling, R. L. C., Wiersema, K., Levan, A. J., et al. 2011, MNRAS, 411, 2792

Stritzinger, M., Mazzali, P., Phillips, M. M., et al. 2009, ApJ, 696, 713

Thöne, C. C., Michałowski, M. J., Leloudas, G., et al. 2009, ApJ, 698, 1307

Tremonti, C. A., Heckman, T. M., Kauffmann, G., et al. 2004, ApJ, 613, 898

Tully, R. B., Rizzi, L., Shaya, E. J., et al. 2009, AJ, 138, 323

van Dokkum, P. G. 2001, PASP, 113, 1420

van Zee, L., Salzer, J. J., Haynes, M. P., O’Donoghue, A. A., \& Balonek, T. J. 1998, AJ, 116, 2805

Vink, J. S., \& de Koter, A. 2005, A\&A, 442, 587

Wiersema, K., Savaglio, S., Vreeswijk, P. M., et al. 2007, A\&A, 464, 529

Woosley, S. E., \& Heger, A. 2006, ApJ, 637, 914

Woosley, S. E., Langer, N., \& Weaver, T. A. 1993, ApJ, 411, 823

Yoon, S., \& Langer, N. 2005, A\&A, 443, 643

Yoon, S., Woosley, S. E., \& Langer, N. 2010, ApJ, 725, 940

Zackrisson, E., Bergvall, N., Olofsson, K., \& Siebert, A. 2001, A\&A, 375, 814

Zaritsky, D., Kennicutt, Jr., R. C., \& Huchra, J. P. 1994, ApJ, 420, 87 